\title{
Corrosion Properties of AISI 4140 Industrial Steels Coated with Aniline in Different Storage Conditions
}

\author{
İ.H. KARAHAN* \\ Mustafa Kemal University, Faculty of Art and Science, Department of Physics, 31040 Hatay, Turkey \\ Polyaniline (PANI) was successfully deposited by means of cyclic voltammetry technique from $0.10 \mathrm{M}$ ani- \\ line containing $0.30 \mathrm{M}$ oxalic acid and $1 \mathrm{~g} / \mathrm{l} \mathrm{H}_{3} \mathrm{BO}_{3}$ solution on AISI 4140 steel electrode. In this investigation \\ nominate scan rates $25,50,75$, and $100 \mathrm{mV} / \mathrm{s}$ have been examined. PANI film was characterized using optical \\ microscope; it was covered with a dark green-yellow homopolymer film of strongly adherent homogeneous char- \\ acteristic. The corrosion behaviour of the steel electrodes with and without PANI film in $3.5 \% \mathrm{NaCl}$ solution \\ was investigated through anodic polarization curves. The results indicated that PANI coating led to decrease of \\ the permeability of metallic plating. The PANI homopolymer film provided an effective barrier property and a \\ remarkable anodic protection to substrate.
}

DOI: 10.12693 /APhysPolA.130.286

PACS/topics: $82.35 . \mathrm{Gh}, 81.65 . \mathrm{Kn}$

\section{Introduction}

Synthesis of conducting polymers onto active metals via electrodeposition has been a subject of many researches due to their attractive corrosion protection properties of these coatings. A successful electropolymerization requires the formation of a passive layer, which might be able to inhibit the dissolution of the oxidizable metal without blocking the access of the monomer and its further oxidation. PANI and its derivatives have been widely studied due to the low potential for polymer formation, good environmental stability, easy to synthesis, controllable electric conductivity, simplicity in doping and dedoping, stability of the formed films, mechanical flexibility [1-3], and high specific capacitance [4-6].

In contrast to classical varnish layers, the polymer offers active protection by exchanging electrons with the metallic substrate. The protection arises from the oxidation or passivation of iron, the steel corrosion potential is placed at more positive values, and the transition from an oxidized to a reduced form adjusts the oxygen reduction reaction in acidic medium. PANI is actually a class of polymers differentiated from one to another by the number of quinoid rings $(\mathrm{Q}$, from 0 to 2) in the elementary unit of four rings, the other rings being benzenoid (B). The particular insulating forms are leucoemeraldine base LB (4B), emeraldine base $\mathrm{EB}(3 \mathrm{~B}, 1 \mathrm{Q})$, and pernigraniline base $\mathrm{PB}(2 \mathrm{~B}, 2 \mathrm{Q})$. In acid or oxidizing environment, the amine sites are easily protonated. The importance of these materials initiates in the existence of a conducting form: emeraldine salt, ES. Either oxidation of LB or protonation of EB lead to ES, which can present two possible organizations. One is polaron lattice (called also semiquinone) wherein the

*e-mail: ihkarahan@gmail.com delocalization of charges allows the conductivity to reach metallic values; the other form is bipolaron. Throughout the electrochemical polarization of PANI, LB is stable at negative potentials. When the potential increases, the progressive charge of LB is first observed, up to two polaronic sites per elementary unit, delocalized in a semiquinone configuration. The conductivity is then highest, and with the potential increase the reorganization of the two polarons in bipolaron corresponds to a conductivity decrease, up to the complete deprotonation in EB. Pernigraniline is steady at very positive potentials only. The polymers are consequently distinguished similarly by their oxidation levels as by their protonation levels; the passageway from one to another includes complex reactions. During an electrochemical polarization, intermediate configurations with a number of charges per unit between 0 and 2, or coexistence of different kinds of units on the polymer backbone are commonly found [7].

The main aim of the current study is the electrosynthesis of PANI on the bare AISI 4140 steel in $0.10 \mathrm{M}$ aniline, $0.30 \mathrm{M}$ oxalic acid and $1 \mathrm{~g} / \mathrm{H}_{3} \mathrm{BO}_{3}$ containing solution and characterization of the polymer coated electrodes. It was also proposed to compare some fundamental characteristics (such as, electrochemical behavior, electrocatalytic activity and stability) of the PANI deposited on bare steel electrodes. The effect of the scan rate on the corrosion properties of PANI coated AISI 4140 electrode was also investigated using cyclic voltammetry technique.

\section{Experimental}

All electrochemical experiments were performed in a standard one-compartment three-electrode cell. The reference electrode was $\mathrm{Ag} / \mathrm{AgCl}(3 \mathrm{M}, \mathrm{KCl})$ and the counter electrode was a platinum wire. In this study, all electrode potential values were referred to the $\mathrm{Ag} / \mathrm{AgCl}$ reference electrode. Moreover, the working electrode used in this study was AISI 4140 steel disks with the 
composition: $\quad 0.36 \mathrm{C}-0.80 \mathrm{Mn}-0.005 \mathrm{Si}-0.914 \mathrm{Cr}-0.30 \mathrm{Ni}-$ $0.85 \mathrm{Mo}-0.075 \mathrm{~V}-0.07 \mathrm{~S}-0.143 \mathrm{Cu}-0.034 \mathrm{P}$ (wt\%). The surface of this electrode was carefully polished with sand paper (1200 grid), degreased with $1 / 1$ ethanol/acetone mixture, washed with deionized water and finally dried by air. Cyclic voltammetry (CV) was performed by scanning from -0.5 to $+1.5 \mathrm{~V}$ followed by reversing the scan to $-0.5 \mathrm{~V}$ vs. $\mathrm{Ag} / \mathrm{AgCl}$ reference electrode. Nominate scan rates $25,50,75$, and $100 \mathrm{mV} / \mathrm{s}$ have been examined.

PARSTAT 2273 electrochemical analyser/work station was utilized for all electrochemical experiments. PANI film was synthesized electrochemically using cyclic voltammetry technique. The anodic polarization curves were recorded in corrosive test solution. The scan rate used in all experiments was $10 \mathrm{mV} / \mathrm{s}$ and the measured open circuit potential value was the initial potential for this scan rate. Additionally, PANI film was analysed through the Fourier transform infrared (FTIR) spectral peaks (Perkin Elmer spectrum 65 model with universal attenuated total reflectance (ATR) attachment with diamond).

Throughout electro-polymerization, the electrolyte contained $0.3 \mathrm{M}$ oxalic acid, $0.1 \mathrm{~g} / 1$ boric acid and $0.1 \mathrm{M}$ aniline. The solutions were prepared as following: first diluting oxalic acid in doubly deionized water, then aniline was slowly added to the mixture. Noticeably, the aniline was used as-received and stored in a dark bottle. The aniline-oxalic acid solution formed white crystals, which re-dissolved after about $45 \mathrm{~min}$ of mixing. Significantly, all studies were done at ambient temperature.

\section{Results and discussion}

Figure 1 shows the three oxidation peaks of PANI on steel obtained from the CV studies during the electropolymerization. The first peak starts at approximately

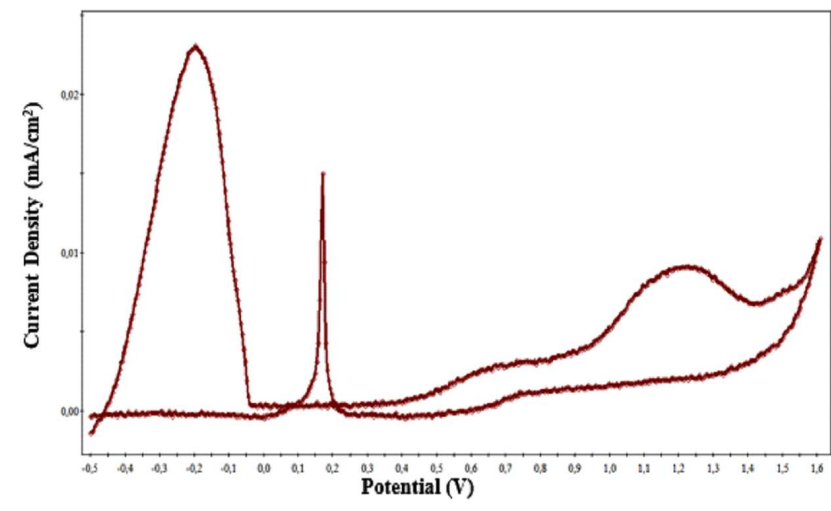

Fig. 1. Cyclic voltammograms of electropolymerization of PANI on AISI 4140 steel at a scan rate of $10 \mathrm{mV} / \mathrm{s}$.

$-0.5 \mathrm{~V}$ vs. $\mathrm{Ag} / \mathrm{AgCl}$ reaching a limiting current density of about $0.023 \mathrm{~mA} / \mathrm{cm}^{2}$ at $-0.25 \mathrm{~V}$ at a scan rate of $10 \mathrm{mV} / \mathrm{s}$. Remarkably, the potential where the current density starts to rise at about $-0.5 \mathrm{~V}$ corresponds to the dissolution of metallic iron to ferrous ions [8]. With sustained polarization, a significant increase in current density is observed between $+0.2,+1.2$, and $+1.5 \mathrm{~V}$, while minor breakers can be seen at $+0.60 \mathrm{~V}$. Essentially, at approximately $+1.4 \mathrm{~V}$ the current increases sharply and the green emeraldine form of PANI is observed on the steel substrate. After reaching a maximum, the current falls sharply. This sharp decrease on the positive side of the wave is likely due to complete passivation of the electrode surface by the formation of an $\mathrm{Fe}^{2+}$ oxalate complex.

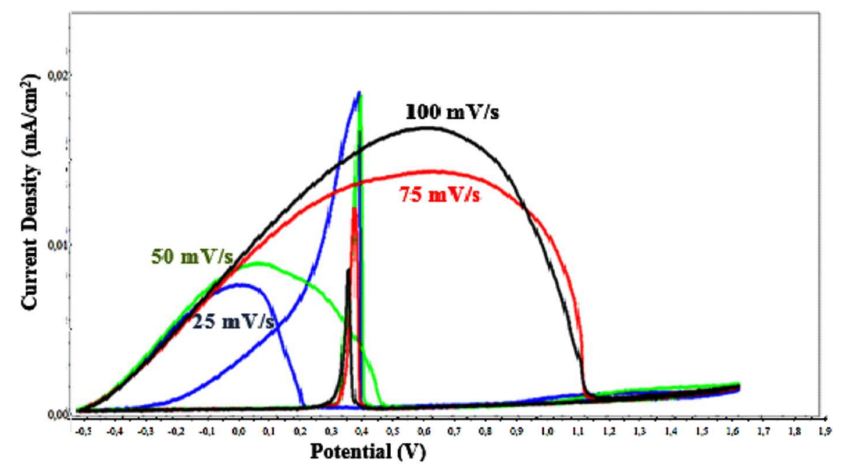

Fig. 2. Cyclic voltammograms for different scan rate values.

Analogously with the increase in the peak current $\left(I_{\mathrm{p}}\right)$ there is a shift in the potential $\left(E_{\mathrm{p}}\right)$ in the anodic direction. This passivation layer does not form at high sweep rates (i.e. above $25 \mathrm{mV} / \mathrm{s}$ ) likewise no well-defined anodic wave was seen in the CV traces. Therefore, to deposit a layer of iron oxalate prior to deposition of PANI requires a scan rate less than $25 \mathrm{mV} / \mathrm{s}$ (Fig. 2).

In the typical emeraldine PANI film, there are 16 approximately equivalent carbons, six carbon-nitrogen single bonds and two carbon-nitrogen double bonds. This ratio of three carbon species is $8: 3: 1$.
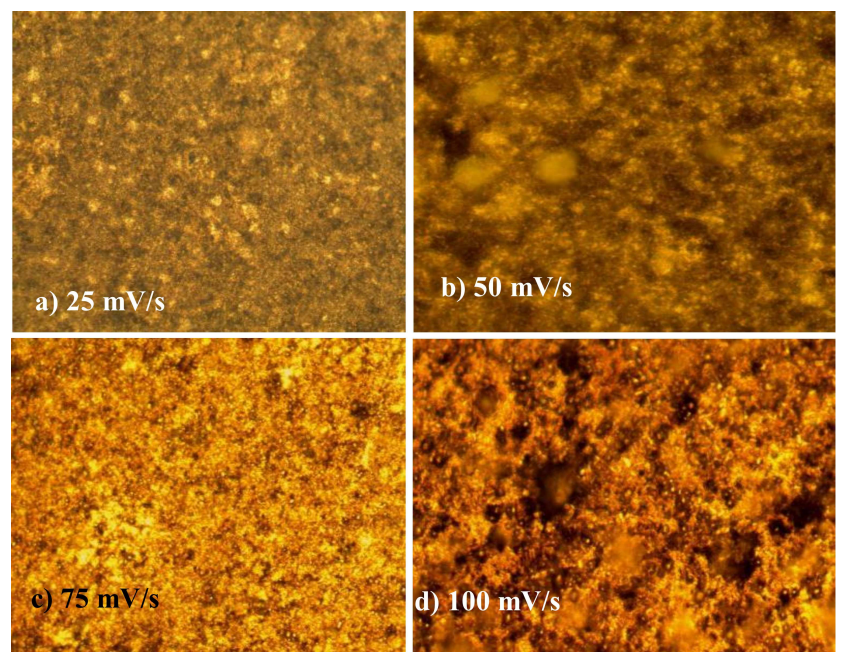

Fig. 3. Optical microscope images of the PANI films electrodeposited at different scan rate: (a) $25 \mathrm{mV} / \mathrm{s}$, (b) $50 \mathrm{mV} / \mathrm{s}$, (c) $75 \mathrm{mV} / \mathrm{s}$, and (d) $100 \mathrm{mV} / \mathrm{s}$. 
The optical microscope images of the PANI films on a 4140 steel substrate deposited at different scan rates are shown in Fig. 3. PANI deposits can be synthesised very uniformly, where higher scan rates present a limited number of relatively large crystals. Moreover, increasing scan rates led to increase in the grain sizes and number of holes of the films.

Another attempt was investigating the effect of scan rate on the corrosion of the electrodeposited PANI films in $3.5 \mathrm{wt} \% \mathrm{NaCl}$ aqueous solution at room temperature. Figure 4 shows the potentiodynamic polarization curves of the PANI coated AISI 4140 steel at room temperature at different scan rates. The range of scan rate was varied from 25 to $100 \mathrm{mV} / \mathrm{s}$. It was observed that the corrosion rates of the samples extended to active potentials with higher scan rates. At the anodic part of the Tafel plot the current started to increase slightly after the peak. The current tends to a limited value until a potential reached $-300 \mathrm{mV}$. It is also observed that increasing the scan rate gradually from 25 to $100 \mathrm{mV} / \mathrm{s}$ leads to a decrease in the anodic current values. The effect of changing the scan rate has higher impact on the anodic process than the cathodic process. It can be concluded that increase in the scan rate leads to increase in the critical current.

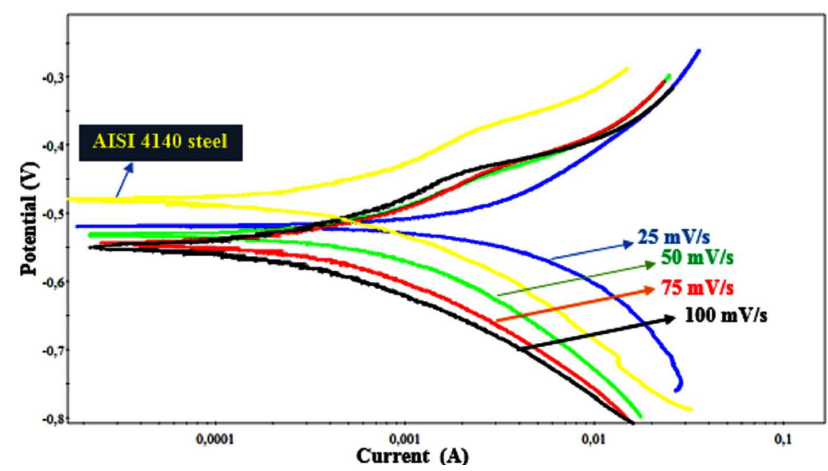

Fig. 4. Tafel plot showing the influence of the scan rate on corrosion properties of PANI coated AISI 4140 steel in a $3.5 \mathrm{wt} \% \mathrm{NaCl}$ aqueous solution.

Table I includes the electrochemical parameters for PANI in $3.5 \mathrm{wt} \% \mathrm{NaCl}$. It can be seen that the opencircuit potential $\left(E_{\mathrm{oc}}\right)$ shifts toward the negative direction with increase in the scan rates. In addition, increase in the scan rate leads to decrease in the corrosion current $\left(I_{\text {corr }}\right)$. An anodic and cathodic Tafel constant ( $\beta_{\mathrm{a}}$ and $\beta_{\mathrm{c}}$, respectively) decreases with increase in the scan rates. It can be concluded that the best scan rate is $25 \mathrm{mV} / \mathrm{s}$ since at this value the corrosion rate is very low (2.166 mpy) in comparison to other PANI coated steels.
TABLE I

The electrochemical parameters for PANI in 3.5wt\% NaCl.

\begin{tabular}{c|c|c|c|c|c}
\hline \hline $\begin{array}{c}\text { Scan rate } \\
{[\mathrm{mV} / \mathrm{s}]}\end{array}$ & $\begin{array}{c}E_{\mathrm{oc}} \\
{[\mathrm{mV}]}\end{array}$ & $\begin{array}{c}I_{\text {corr }} \\
{\left[\mu \mathrm{A} / \mathrm{cm}^{2}\right]}\end{array}$ & $\begin{array}{c}\beta_{\mathrm{c}} \\
{[\mathrm{mV}]}\end{array}$ & $\begin{array}{c}\beta_{\mathrm{a}} \\
{[\mathrm{mV}]}\end{array}$ & $\begin{array}{c}\text { Corrosion } \\
\text { rate }[\mathrm{mpy}]\end{array}$ \\
\hline 25 & -518.661 & 728.5 & 337.701 & 374.041 & 2.166 \\
50 & -532.406 & 747.3 & 178.269 & 143.704 & 2.222 \\
75 & -544.009 & 504.7 & 165.104 & 133.990 & 15.00 \\
100 & -549.499 & 194.9 & 127.130 & 102.718 & 57.95
\end{tabular}

\section{Conclusion}

Polymerization of aniline from an oxalic acid medium is accompanied by the passivation of steel. An iron(II) oxalate coating is formed first which is further oxidized to an iron(III) species at positive potentials. Aniline polymerizes on this iron(III) layer forming predominately the emeraldine salt. Reversing the scan reduces the iron(III) coating to the iron(II) species and this is necessary to achieve good adhesion of PANI to steel. The kinetics of passivation is crucial for obtaining good adhesion of the PANI coating. The effect of scan rate on the corrosion of the electrodeposited PANI films was investigated. The scan rate $25 \mathrm{mV} / \mathrm{s}$ was the best scan rate because at this scan rate the corrosion rate is very low compared to other PANI coated steels.

\section{References}

[1] Y. Cao, P. Smith, A.J. Heeger, Synth. Met. 57, 3514 (1993).

[2] D.S. Patil, J.S. Shaikh, D.S. Dalavi, M.M. Karanjkar, R.S. Devan, Y.R. Ma, P.S. Patil, J. Electrochem. Soc. 158, A653 (2011).

[3] D.S. Patil, S.A. Pawar, R.S. Devan, Y.R. Ma, W.R. Bae, J.H. Kim, P.S. Patil, Mater. Lett. 117, 248 (2014).

[4] X. Zhou, L. Li, S. Dong, X. Chen, P. Han, H. Xu, J. Yao, C. Shang, Z. Liu, G. Cui, J. Solid State Electrochem. 16, 877 (2012).

[5] S. Dhibar, S. Sahoo, C.K. Das, R. Singh, J. Mater. Sci. Mater. Electron. 24, 576 (2013).

[6] X. Xiang, E. Liu, L. Li, Y. Yang, H. Shen, Z. Huang, Y. Tian, J. Solid State Electrochem. 15, 579 (2011).

[7] M.C. Bernard, S. Joiret, A. Hugot-Le Goff, P.V. Phong, Electrochem. Soc. 148, B12 (2001).

[8] M. Pourbaix, Proceedings of the Atlas of Electrochemical Equilibria in Aqueous Solutions, Pergamon Press, New York 1966. 